\title{
The Coaching Behaviour Scale for Sport (CBS-S): A psychometric evaluation of the Swedish version
}

Andreas Carlsson and Carolina Lundqvist

The self-archived postprint version of this journal article is available at Linköping University Institutional Repository (DiVA):

http:/ / urn.kb.se/ resolve?urn=urn:nbn:se:liu:diva-153207

N.B.: When citing this work, cite the original publication.

Carlsson, A., Lundqvist, C., (2016), The Coaching Behaviour Scale for Sport (CBS-S): A psychometric evaluation of the Swedish version, Scandinavian J ournal of Medicine and Science in Sports, 26(1), 116-123. https:// doi.org/ 10.1111/ sms.12359

Original publication available at:

https:/ / doi.org/ 10.1111/sms.12359

Copyright: J ohn Wiley and Sons

http:// www.wiley.com/ 
The Coaching Behaviour Scale for Sport (CBS-S):

A Psychometric Evaluation of the Swedish Version

\author{
Accepted version \\ Andreas Carlsson \& Carolina Lundqvist \\ The Swedish School of Sport and Health Sciences
}

Author Note

Correspondence concerning this article should be addressed to Carolina Lundqvist, The Swedish School of Sport and Health Sciences, Box 5626, SE-114 86 Stockholm. E-mail: carolina.lundqvist@gih.se. 


\begin{abstract}
The present study validated a Swedish version of the 47-item Coaching Behaviour Scale for Sport (CBS-S). Sample 1 consisted of 506 team sport athletes (262 men and 244 women; mean age: $22.20, S D=3.90$ ) distributed across 41 coaches at the two highest national levels of various sports. Athletes completed the CBS-S and established questionnaires of coaching behaviours (LSS), self-confidence (CSAI-2R) and coach-athlete relationship (CART-Q). An additional sample of 39 basketball players (21 men and 18 women; mean age $=17.40, S D=$ 2.39) completed the CBS-S twice, approximately four weeks apart. Confirmatory factor analysis showed an acceptable model fit for the seven-factor version of the CBS-S, although two items of the negative personal rapport subscale displayed insufficient factor loadings. Correlations between the subscales of the CBS-S and established instruments were in accordance with theoretical expectations, supporting the concurrent validity. Cronbach's alpha (>.82) for all dimensions provided support for the reliability of the CBS-S, and testretest correlations indicated moderate stability over time. Cultural differences in the assessment of coaching behaviours and the usability of the CBS-S by coaches for selfreflection and development are discussed.
\end{abstract}

Keywords: coaching, scale, measurement, coach development, team sport athletes 


\section{Introduction}

The coach plays a pivotal role in athletes’ sport execution, and various coach behaviours can affect athletes positively or negatively. For example, some behaviour may help reduce anxiety, increase self-confidence and the desire to continue participation, and enhance skill development (Hays et al., 2007; Smith \& Smoll, 2007; Becker, 2009). Other coach behaviours may induce anger, distractions, team divisions and demotivation (Gearity \& Murray, 2011). Moreover, coaching behaviours will ultimately also impact the quality and type of relationship between coach and athlete, which is a central part of the coaching process (Jowett \& Chaundy, 2004). However, coaches and athletes often report disparate opinions about the coach behaviours actually used (Smith \& Smoll, 2007), and studies have revealed that coaches also may be unaware of their own behaviours or may overestimate the frequency of their positive behaviours (Millar et al., 2011; Partington \& Cushion, 2011). Self-reflection and self-awareness of one's own behaviours are suggested as important characteristics of expert coaches (Schempp et al., 2006), and a lack thereof might induce difficulties in a coach's professional development and accentuate the risk of adopting inappropriate coaching behaviours. For coaches, behavioural feedback is argued to be one of the most powerful ways to adhere to and improve upon sound coaching practice (Smith \& Smoll, 2007). By getting behavioural feedback from athletes, a coach could also stand to improve the congruence in the coach-athlete relationship, which is deemed important (Lorimer \& Jowett, 2009). However, inherently it is somewhat difficult for sports coaches to get behavioural feedback on their coaching practice (cf. Dunn \& Shriner, 1999). Research has repeatedly highlighted coaches’ low ability to read athletes’ states and preferences (Vargas-Tonsing \& Guan, 2007; Lorimer \& Jowett, 2009), thus making it difficult for the coaches to get a read of the athletes' opinions. Assessments by which athletes provide feedback on a coach's behaviours may help stimulate 
the coach's self-reflection in order to purposefully work to modify and improve positive coaching behaviours for the athletes at hand. Thus, through applied instruments coaches can receive feedback from their athletes and make an informed decision regarding what areas need development (Mallett \& Côté, 2006).

One of the most popular scales for assessing coaching behaviours to date is the Leadership Scale in Sports (LSS; Chelladurai \& Saleh, 1980). The psychometric qualities of this scale have been questioned, however (e.g., Chelladurai, 1990; 2007), and attempts to revise and improve it have been only modestly successful (Jambor \& Zhang, 1997). The Coaching Behaviour Scale for Sport (CBS-S; Côté et al., 1999) has been suggested as a more comprehensive measure of coaching behaviour as it also captures important parts of coaching, such as mental preparation and competition strategies, not covered by the LSS. The CBS-S was developed based on the behavioural dimensions of the Coaching Model (CM; Côté et al., 1995b). Thus, items in the CBS-S were created from the CM components (a) training, for example the coach’s interaction style, ability to make practice competition-like, and knowledge of technical and mental skills; (b) competition, including routines and behaviours at the competition site; and (c) organization in cooperating with parents and coaching staff, creating training plans and helping athletes with their personal concerns (Côté et al., 1995a). In previous studies the CBS-S has shown good reliability and concurrent validity, and has been used to study the influence of coaching behaviours on athletes' anxiety (Baker et al., 2000) and the development of life skills in former high school athletes (Gould \& Carson, 2010), and to investigate satisfaction in team sport athletes and individual sport athletes (Baker et al., 2003). Slightly different versions of the CBS-S have been used in these studies; therefore the number of items has varied from 37 up to the present 47. Predominantly, the number of items in the subscale of negative personal rapport has varied in different versions of the scale, increasing from three initially to the present seven (Côté, J, personal 
communication). Moreover, a subscale labelled competition strategies, not included in the initial study (Côté et al., 1999), was later added (Baker et al., 2000). To date, only one study has used confirmatory factor analysis (CFA) to confirm the proposed factor structure of the 47-item version of the scale (Koh et al., in press). Koh and colleagues decided, however, to remove one item of the CBS-S competition strategies subscale which was argued to be irrelevant for the specific sample under study. Their findings supported the factorial validity of the 46 remaining items, and also the concurrent validity of the CBS-S with coaching satisfaction (Koh et al., in press).

CBS-S could prove beneficial in the development of good coaching practice, as has been suggested (Mallet \& Côté , 2006) and shown in a recent study using the English version of the CBS-S (Koh et al., submitted). In line with the vast majority of existing coaching research conducted (Cushion, 2010), the CBS-S was developed for and evaluated among North American coaches and athletes. Sporting systems and cultures may vary across countries; thus the results or assessments cannot automatically be generalized to other cultures (Kline, 2009; Cushion, 2010). The original English version of the CBS-S has not yet been translated into or empirically evaluated in other languages. Thus, there is a need to carefully validate it in various sport systems and cultures to further establish its generalizability and usefulness in various countries and contexts.

The aim of the present study was to evaluate the psychometric properties of a translated Swedish version of the CBS-S in terms of factorial validity, concurrent validity, internal consistency and test-retest reliability among team sport participants. In terms of factorial validity, the original correlated seven-factor model was tested, which includes the latent variables: physical training and planning, technical skills, goal setting, mental preparation, competition strategies, personal rapport and negative personal rapport. In order to evaluate the concurrent validity, three hypotheses were formulated in terms of expected associations of the 
CBS-S and assessments of coach behaviours/leadership, self-confidence and coach-athlete relationship: (a) The dimensions in the CBS-S and LSS both approach coaching behaviours and leadership, but from partly different angles. For example, the LSS arranges instruction and feedback as separate dimensions while the CBS-S includes these features indirectly in many of the subscales. Thus, it was expected that the four positive leadership dimensions covered in the LSS (training and instruction, democratic behaviour, social support, and positive feedback) should display moderate positive correlations with all CBS-S subscales, except for with negative personal rapport, which was expected to display negative correlations. (b) Athletes' self-confidence has been proposed as an important outcome of coaching behaviours in the updated Coaching Model (Côté et al., 2010), but it is also known that an athlete's self-confidence is influenced by a number of sources (e.g., performance accomplishments and experience; Hays et al., 2007). Thus, it was expected that selfconfidence would show weak to moderate positive correlations with all subscales of the CBSS, except for negative personal rapport, which was expected to show negative correlations. (c) The athlete-coach relationship is an integral part of coaching and coaching behaviours used (Jowett \& Chaundy, 2004); thus moderate to large correlations were expected between the CBS-S subscales and assessments of coach-athlete relationships. Negative personal rapport was the only CBS-S subscale with an expected negative association. Particularly the CBS-S subscale of positive rapport, which includes items directly related to the consideration of the athlete, was expected to display a strong positive correlation with assessments of coachathlete relationships.

\section{Materials and Methods}

\section{Participants and Procedures}

First, in order to collect a large sample of team athletes (Sample 1) a total of 58 team coaches of basketball, floor ball, soccer, team handball and volleyball, all teams competing at 
the two highest national levels in Sweden, were contacted and given information about the purpose of the study and ethical information. A total of 49 coaches agreed to participate in the study, but for logistical reasons (e.g., training schedules or the fact that the team's competitive season ended before data collection could be undertaken) eight teams were excluded. Thus, 71\% (21 men’s teams and 20 women’s teams) of the originally contacted coaches were included in the study. From the teams, a total of 506 athletes (262 men and 244 women) with a mean age of $22.20(S D=3.90)$ volunteered to participate (basketball: $n=191$, floor ball: $n=128$, soccer: $n=23$, team handball: $n=174$ and volleyball: $n=29)$ and complete the CBS-S and additional inventories for concurrent validation analyses of the CBS-S. The participants practiced in average 4.28 times a week $(S D=1.38)$ with their head coach, and had been coached by their present head coach for 2.51 years $(S D=2.36)$. Seven participants returned incomplete or carelessly filled inventories to the point that the responses were unusable, reducing the sample to 499 participants. Secondly, an additional independent sample (Sample 2) of 39 of the basketball players (21 men and 18 women; mean age $=17.40, S D=2.39$ ) agreed to complete the CBS-S twice, approximately four weeks apart. On average, the participants in the second sample practiced 4.20 times a week $(S D=1.49)$ with their head coach, and had been coached by their present head coach for 2.10 years $(S D=1.55)$.

To avoid potential influence from competition, the surveys were collected in connection with practice or a team event other than competition. Before completing the questionnaires, the athletes received the same verbal and written information as the coaches were initially provided, and signed written informed consent. All procedures in the study were approved by the regional ethical board (No. 2012/2152-31/5) in accordance with Swedish national ethical standards.

\section{Measurements}


The 47-item CBS-S measures six dimensions of positive coaching behaviours physical training and planning (7 items, e.g., "provides me with a plan for my physical preparation”, total score range: 7-49), technical skills (8 items, e.g., “gives me reinforcement about correct technique”, total score range: 8-56), goal-setting (6 items, e.g., “helps me set long-term goals”, total score range: 6-42), mental preparation (5 items, e.g., "provides advice on how to perform under pressure”, total score range: 5-35), competition strategies (7 items, e.g., “keeps me focused in competitions”, total score range: 7-49), and personal rapport (6 items, e.g., “is a good listener”, total score range: 6-42) - and one dimension of negative coaching behaviours: negative personal rapport (8 items, e.g., “intimidates me physically”, total score range: 8-56). All items begin with the stem "My coach..." and are rated on a seven-point scale ranging from 1 (never) to 7 (always). The CBS-S was translated into Swedish by use of back-translation (Brislin, 1970). The English version was initially translated into Swedish by the first author, and was then examined by the second author, resulting in minor changes. Secondly, an independent coach completed the translated CBS-S by use of a think-aloud procedure (Jääskeläinen, 2010), and the information provided through the coach's interpretations of items resulted in minor adjustments to increase the clarity of the items. The next step involved an independent bilingual researcher unfamiliar with the CBS-S back-translating the Swedish version to English, resulting in few adjustments. Finally, a pilot study was carried out with 24 team sport athletes, who completed the scale and at the same time were urged to note any ambiguities in any item. No changes were made after this pilot study.

To examine the concurrent validity of the CBS-S three well-validated scales, which all have been psychometrically examined in their Swedish versions (cf. Chelladurai, 1990, Lundqvist \& Hassmén, 2005; Yang \& Jowett, 2011), were included in the survey: 
(a) A Swedish version of the 40-item Leadership Scale in Sport (LSS; Chelladurai \& Saleh, 1980; Chelladurai, 1990), measuring five subscales of coaching behaviours (training and instruction (total score range: 13-65), social support (total score range: 8-40), positive feedback (total score range: 5-25), democratic behaviour (total score range: 9-45) and autocratic behaviour (total score range: 5-25)). Because the autocratic subscale has displayed unsatisfactory internal consistency in previous research (Chelladurai, 2007), this subscale was not utilized in the present study. Respondents rate their answers on a five-point Likert scale ranging from 1 (never) to 5 (always).

(b) The coach-athlete relationship was measured using a Swedish version of the CoachAthlete Relationship Questionnaire (CART-Q; Jowett \& Ntoumanis, 2004; Yang \& Jowett, 2011). The CART-Q includes 11 items, rated on a seven-point Likert scale ranging from 1 (strongly disagree) to 7 (strongly agree), which assess closeness, engagement and complimentarily. In this study, one aggregate score of the three subscales was computed (total score range: $11-77)$.

(c) Self-confidence was measured using the self-confidence subscale in the Swedish version of Competitive State Anxiety Inventory-2 (CSAI-2R; Cox et al., 2003; Lundqvist \& Hassmén, 2005). The subscale consists of five items rated on a four-point Likert scale ranging from 1 (not at all) to 4 (very much) (total score range: 5-20).

\section{Statistical Analyses}

Sample 1 was used to evaluate the factorial validity of the CBS-S by use of confirmatory factor analyses (CFA) with robust weighted least square estimation (RWLS) performed in Mplus 7 (Muthén \& Muthén, 1998-2012). No multivariate outliers of the CBS-S were identified (Mahalanobis distance: $p<.001$ ). Missing values related to the CBS-S represented a total amount of $0.05 \%$ of all responses. In the CFA analysis, missing responses were estimated as a function of the covariates available as an option in Mplus when RWLS is used. 
Goodness of fit was evaluated using the chi-square $\left(\chi^{2}\right)$, the comparative fit index (CFI), the Tucker-Lewis index (TLI), and the root mean square error of approximation (RMSEA) including the $90 \%$ confidence interval (90\% CI). A small and nonsignificant $\chi^{2}$ indicates a good model fit, but is also known to be highly sensitive to sample size (Bollen, 1989). The CFI and TLI are generally accepted to indicate a reasonable fit at a value of .90 and a close fit at values .95 or above (Tabachnick \& Fidell, 2007; Hair et al., 2014). Regarding the RMSEA, values close to or less than .05 are commonly regarded to indicate a good fit, and values less than .08 an acceptable fit (Hair et al., 2014). Although the above cut-off values have been evaluated based on continuous data, Yu (2002) showed that they are reasonable to use also with categorical data. Sample 1 was also used to evaluate the Cronbach's alpha of the CBS-S subscales and the Pearson product moment correlations with external and related variables, and these analyses were performed by use of the SPSS 21.0. Of the total amount of data collected in Sample 1, missing values constituted 1.8\% of all responses, and these values were imputated by expectation minimization (EM; Hair et al., 2014). When the CBS-S and the external variables were screened for multivariate outliers (Mahalanobis distance, $p<0.001$ ), four cases were identified and removed from further analyses. Thus, the effective sample in the construct validation analysis constituted 495 cases. Finally, Sample 2 was utilized in order to evaluate the test-retest reliability of the CBS-S by use of Pearson product moment correlations in the SPSS 21.0. A total of .25\% of all responses in Sample 2 constituted missing values, and these were imputated using EM (Hair et al., 2014). Mahalanobis distance $(p<.001)$ identified no multivariate outliers in Sample 2.

\section{Results}

\section{Descriptives and Confirmatory Factor Analysis}


Skewness and kurtosis, inter-factor correlations of total scores of the CBS-S subscales, mean values and standard deviations for Sample 1 are displayed in Table 1. Cronbach's alpha scores for all subscales are also shown, indicating adequate internal consistency $(\alpha>.70)$ in all dimensions. The a priori specified model tested was the original seven-factor correlated model. Results showed the chi-square statistics to be significant $\left(\chi^{2}(1013)=3307.82, p<\right.$ .001 ), but goodness of fit indices indicated an acceptable model fit (CFI $=.95$, TLI $=.94$, RMSEA $=.067(90 \%$ CI $=.065-.070)$. The standardized solution of factor loadings, error estimates, explained variance and error variance are displayed in Table 2, and the results indicated appropriate factor loadings for all items in the six positive coaching behavioural subscales. Two items in the subscale of negative personal rapport were identified with insufficient factor loadings (i.e., item 40: “uses fear in his/her coaching methods" and item 41: "yells at me when angry"), suggesting the items to be weaker indicators of the latent factor. In addition, a third item of this subscale (item 44: “intimidates me physically") contributed marginally to the latent factor.

\section{Test-Retest Reliability and Concurrent Validity}

As shown in Table 3, analyses of the test-retest reliability showed satisfactory stability over time for the subscales of physical training and planning, personal rapport and negative personal rapport ( $r$ range: .75-.82). However, the stability was questionable for the remaining subscales ( $r$ range: .58-.66). Investigation of the concurrent validity, also displayed in Table 3, revealed that the positive coaching behaviour subscales of the CBS-S (i.e., physical training and planning, technical skills, mental preparation, goal-setting, competition strategies and personal rapport) all displayed significant and positive correlations to the positive leadership dimensions assessed by the LSS (i.e., training and instruction, democratic behaviour, social support and positive feedback). The associations between the CBS-S subscale of physical 
training and planning and the LSS subscales were relatively weak, however ( $r$ range: .13-.34). Physical training and planning was also the only dimension which did not show any significant association with assessments of self-confidence. The remaining CBS-S subscales displayed significant and rather weak correlations to self-confidence in the expected directions. The same trend was also shown in the correlations between the CBS-S subscales and the coach-athlete relationship assessed by the CART-Q, where physical training and planning displayed a somewhat weaker positive relation to the CART-Q than the remaining

CBS-S dimensions. As expected, personal rapport and CART-Q displayed a strong association. In line with the hypotheses, negative personal rapport showed a negative correlation to all external variables assessed.

\section{Discussion}

The purpose of the present study was to examine the psychometric properties of the Swedish version of the 47-item version of CBS-S. The results of this study provide overall support for the translated version of the scale in terms of factorial and concurrent validity as well as the internal consistency. The test-retest coefficients indicated questionable stability of four of the seven subscales of the CBS-S (i.e., mental preparation, technical skills, goal setting and competition strategies). The results found in the present study are nevertheless comparable to the test-rest correlations reported previously when the original English version of the CBS-S was evaluated (Côté et al., 1999). Thus, considering that the CBS-S assesses behaviours which to some extent are likely to fluctuate over time, as well as results previously reported in the literature, the questionable stability found in this study likely refers to the assessed construct rather than to the translation of the scale.

An acceptable model fit was displayed for the seven-factor version of the translated version of the scale. Two items of the negative personal rapport subscale revealed weak factor 
loadings. In the original study of the CBS-S, the negative personal rapport subscale included only three items. This dimension has since been gradually modified and expanded (e.g., Gould \& Carson, 2010 used a version with 44 items in all), which may indicate concern regarding this subscale in previous research. Nevertheless, Koh and colleagues (in press) reported satisfactory factor loadings of all items in the negative personal rapport subscale, which indicates that the weak factor loadings may be related directly to the Swedish version. Whereas it is plausible that these findings are due to problems with the translation of the items, they may also be a consequence of cross-cultural differences. An inspection of the wording of these two items (item 40: "uses fear in his/her coaching methods" and item 41: "yells at me when angry”), along with a third (item 44: “intimidates me physically") which also displayed a substantially lower factor loading compared to the rest of the items in the subscale, shows that they all capture similar content. The content of these items closely resembles what Bartholomew et al. (2010) labelled intimidation in their negative coaching behaviours instrument. Coaching behaviours are influenced by both the sporting systems and the cultures across countries and sports. The Swedish and North American sport traditions differ substantially, with the Swedish tradition oriented around volunteer commitment as opposed to what is more customary in the North American tradition with its emphasis on school-connected sports organizations (Cushion, 2010). Research conducted in non-sport contexts has also found the Nordic culture to be less assertive and leaders in the Nordic countries, in contrast to those in North America, to be less authoritarian, operating in a less hierarchical environment and emphasizing the group over the individual (Northouse, 2013). It is plausible that this pattern might also be found in sport leaders, implying that the typical Swedish coach might be less inclined to use behaviours linked with intimidation and use more cooperating and relationship-oriented behaviours. Thus, intimidating behaviours may not be as prevalent among Swedish coaches compared to North American ones. Whereas the present 
study can only conclude that two items in the negative personal rapport subscale of the Swedish version cannot be supported in terms of their factor loadings, and thus should be removed when the translated version is used, researchers are encouraged to replicate the findings in various contexts and cultures. Cross-cultural evaluations of the CBS-S would enable further insight as to whether the lack of support for the items in the negative personal rapport subscale found in this study is purely a translation issue or if the if the scale in part needs to be adapted to cultural differences.

In terms of concurrent validity, results displayed in general the expected associations in terms of strength and direction with external inventories. However, the subscale of physical training and planning consistently revealed weaker relationships with the LSS leadership dimensions, self-confidence and coach-athlete relationship than the other CBS-S subscales. A possible explanation for this finding is that the items in the Physical training and planning dimension seem to entail some personal distance between athlete and coach compared to the remaining dimensions, which all have closer interaction or interpersonal communication embedded in the item wordings. The findings in the present study are also in line with the results from Koh et al. (in press), who reported a weaker relationship between Physical training and planning and coaching satisfaction in relation to the remaining positive subscales of the CBS-S. Whereas the present study provides some additional insight into the relationship between the CBS-S and various external variables, further validation studies are desirable in which the relationships between coaching behaviours assessed by the CBS-S and theoretically related variables are closely examined.

In summary, the results suggest in general that the Swedish version of the CBS-S provides a broad and psychometrically sound basis for evaluating coaches in all the four areas Janelle and Hillman (2003) deemed necessary for athletic excellence: technical, cognitive, emotional and physical. In contrast to the questionable psychometric results reported for the 
LSS (Chelladurai, 1990; 2007), the present study showed the CBS-S to have good internal consistency and an adequate factor structure even though single items displayed low factor loadings. Test-retest reliability was comparable to the results obtained in the initial study by Côté et al. (1999). Important variables for the athletes, such as self-confidence and relationship with the coach, were also found to display sound relationships with the dimensions of the CBS-S, supporting the concurrent validity of the scale. Overall, the present study adds to the coaching behaviour literature by confirming the psychometric properties of the CBS-S. It should be noted that the present study focused exclusively on validating the CBS-S for Swedish team sport athletes' evaluation of their coaches. Therefore, the scale's usability with individual athletes is still unknown. Future research is therefore encouraged to empirically evaluate the instrument's psychometric properties with individual sports, and also further test its applicability in evaluating and highlighting areas of improvement for coaches in applied settings.

\section{Perspectives}

This study aimed to investigate the validity and reliability of the CBS-S, which was developed to examine athletes’ views of their coach (es). Findings support the Swedish version of the CBS-S as a tool for obtaining coach behavioural data. Consequently, the generated findings could by extension contribute to both theoretical and applied research aiming to expand the evolving database on coaching. Additionally, the CBS-S could aid in further research on coaching development as the use of instruments offers a possibility for coaches to receive feedback on their behaviours, which is often elusive. Improved coaching could by extension result in important outcomes for the athlete, such as decreased anxiety and dropout from sport as well as health benefits, better skill development and improved performance. Although this study may need to be replicated and extended to individual sports athletes, its findings support the notion of team sport coaching as a global phenomenon 
captured reasonably well with the CBS-S. Cross-cultural research is a valuable aspect in deepening the understanding of sports coaching in various cultures.

Acknowledgements: This study was supported by grants from the Swedish National Centre for Sports Research. We would like to thank Dr. Sanna Nordin-Bates at the Swedish School for Sport and Health Sciences for her valuable assistance in the process of backtranslating the scale.

\section{References}

Baker J, Côté J, Hawes R. The relationship between coaching behaviours and sport anxiety in athletes. J Sci Med Sport 2000: 3: 110-119.

Baker J, Yardley J, Côté J. Coach behaviors and athlete satisfaction in team and individual sports. Int J Sport Psychol 2003: 34: 226-239.

Bartholomew K, Ntoumanis N, Thørgersen-Ntoumani C. The controlling interpersonal style in a coaching context: Development and initial validation of a psychometric scale. J Sport Exerc Psychol 2010: 32: 193-221.

Becker AJ. It's not what they do, it's how they do it: Athlete experiences of great coaching. Int J Sports Sci Coach 2009: 4: 93-119.

Bollen KA. Structural equations with latent variables. New York: Wiley, 1989.

Brislin RW. Back-translation for cross-cultural research. J Cross Cult Psychol 1970: 1: 185216.

Chelladurai P. Leadership in sports: A review. Int J Sport Psychol 1990: 21: 328-354.

Chelladurai P. Leadership in sports. In: Tenenbaum G, Eklund RC. eds. Handbook of sport psychology. $3^{\text {rd }}$ edn. New York: John Wiley and Sons, 2007: 113-135. 
Chelladurai P, Saleh SD. Dimensions of leader behavior in sports: Development of a leadership scale. J Sport Psychol 1980: 2: 34-45.

Côté J, Bruner M, Strachan L, Erickson K, Fraser-Thomas J. Athletes’ development and coaching. In Lyle J, Cushion C. eds. Sport coaching: Professionalisation and practice. Oxford, UK: Elsevier, 2010: 63-83.

Côté J, Salmela J, Russell S. The knowledge of high-performance gymnastics coaches: Competition and training and considerations. Sport Psychol 1995a: 9: 76-95.

Côté J, Salmela J, Trudel P, Baria A, Russell S. The Coaching Model: A grounded assessment of expert gymnastics coaches’ knowledge. J Sport Exerc Psychol 1995b: 17: 1-17.

Côté J, Yardley J, Hay J, Sedgwick W, Baker J. An exploratory examination of the Coaching Behavior Scale for Sport. Avante 1999: 5: 82-92.

Cox RH, Martens MP, Russell WD. Measuring anxiety in athletics: The revised Competitive State Anxiety Inventory-2. J Sport Exerc Psychol 2003: 25: 519-533.

Cushion C. Coach behaviour. In: Lyle J, Cushion C. eds. Sport coaching: Professionalisation and practice. Oxford, UK: Elsevier, 2010: 43-61.

Dunn TG, Shriner C. Deliberate practice in teaching: What teachers do for self-improvement. Teach Teacher Educ 1999: 15: 631-651.

Gearity BT, Murray MA. Athletes’ experiences of psychological effects of poor coaching. Psychol Sport Exerc 2011: 12: 213-221.

Gould D, Carson S. The relationship between perceived coaching behaviors and developmental benefits of high school sports participation. Hell J Psychol 2010: 7: 298314.

Hair JF, Black WC, Babin BJ, Anderson RE. Multivariate data analysis. $7^{\text {th }}$ edn. Essex: Pearson Education Limited, 2014. 
Hays K, Maynard I, Thomas O, Bawden M. Sources and types of confidence identified by world class sport performers. J Appl Sport Psychol: 19: 434-456.

Jambor EA, Zhang JJ. Investigating leadership, gender, and coaching level using the revised leadership for sport scale. J Sport Behav 1997: 20: 313-321.

Janelle CM, Hillman CH. Expert performance in sport: Current perspectives and critical issues. In Starkes J, Ericsson KA. eds. Expert performance in sport: Advances in research on sport expertise. Champaign: Human Kinetics, 2003: 19-48.

Jääskeläinen R. Think-aloud protocol. In: Gambier Y, van Doorsaler L. eds. Handbook of translation studies: Volume 1. Amsterdam, NL: John Benjamins Publishing Company, 2010: 371-373.

Jowett S, Chaundy V. An investigation into the impact of coach leadership and coach-athlete relationship on group cohesion. Group Dyn 2004: 8: 302-311.

Jowett S, Ntoumanis N. The coach-athlete relationship questionnaire (CART-Q):

Development and initial validation. Scand J Med Sci Sports 2004: 14: 245-257.

Kline RB. Becoming a behavioral science researcher: A guide to producing research that matters. New York: Guilford Press, 2009.

Koh, KT, Kawabata, M, Mallett, C. The coaching behaviour scale for sport: Factor structure examination for Singaporean youth athletes. Int J Sports Sci Coach. (in press).

Koh KT, Mallett CJ, Wang CKJ, Masato K. Case studies of guided reflection for coaches of high performance basketball. Phys Ed Sport Ped: submitted.

Lorimer R, Jowett S. Empathic accuracy in coach-athlete dyads who participate in team and individual sports. Psychol Sport Exerc 2009: 10: 152-158.

Lundqvist C, Hassmén P. Competitive State Anxiety Inventory-2 (CSAI-2): Evaluating the Swedish version by confirmatory factor analysis. J Sports Sci 2005: 23: 727-736. 
Mallett C, Côté J. Beyond winning and losing: Guidelines for evaluating high performance coaches. Sport Psychol 2006: 20: 213-220.

Millar S-K, Oldham ARH, Donovan M. Coaches’ self-awareness of timing, nature and intent of verbal instructions to athletes. Int J Sports Sci Coach 2011: 6: 503-513.

Muthén LK, Muthén BO. Mplus statistical analysis with latent variables - user’s guide [availabe at: www.statmodel.com], 1998-2012.

Northouse PG. Leadership: Theory and practice. $6^{\text {th }}$ edn. Thousand Oaks: Sage Publication, 2013.

Partington M, Cushion C. An investigation of the practice activities and coaching behaviors of professional top-level youth soccer coaches. Scand J Med Sci Sports 2011: 21: 1-9.

Schempp PG, McCullick BA, Busch CA, Webster C, Mason IS. The self-monitoring of expert sport instructors. Int J Sports Sci Coach 2006: 1: 25-35.

Smith RE, Smoll FL. Social-cognitive approaches to coaching behaviours. In: Jowett S,

Lavallee D. eds. Social psychology in sport. Champaign: Human Kinetics, 2007: 75-90.

Tabachnick BG, Fidell LS. Using multivariate statistics. $5^{\text {th }}$ edn. Needham Heights, MA: Allyn \& Bacon, 2007.

Vargas-Tonsing TM, Guan J. Athletes’ preferences for informational and emotional pre-game speech content. Int J Sports Sci Coach 2007: 2: 171-180.

Yang SX, Jowett S. Psychometric properties of the Coach-Athlete relationship questionnaire (CART-Q) in seven countries. Psychol Sport Exerc 2012: 13: 36-43.

Yu, C.Y. (2002). Evaluation of model fit indices for latent variable models with categorical and continuous outcomes. Unpublished dissertation. [Retrieved September 29, 2014 from http://www.statmodel.com/download/Yudissertation.pdf] 
Table 1.

Inter-Factor Correlations of Total Scores of the CBS-S Subscales, Internal Consistency and Descriptive Statistics of the CBS-S.

\begin{tabular}{|c|c|c|c|c|c|c|c|}
\hline \multirow[b]{2}{*}{ Variable } & \multicolumn{7}{|c|}{ Sample $1(n=499)$} \\
\hline & 1. & 2 & 3. & 4. & 5. & 6. & 7. \\
\hline $\begin{array}{l}\text { 1. Physical training } \\
\text { and planning }\end{array}$ & -- & $.31 * *$ & $.23 * *$ & $.34 * *$ & $.36 * *$ & $.16^{*}$ & .06 \\
\hline 2. Technical skills & & -- & $.61 * *$ & $.64 * *$ & $.69 * *$ & $.50 * *$ & $-.18 *$ \\
\hline 3. Mental preparation & & & -- & $.71 * *$ & $.64 * *$ & $.45^{* *}$ & $-.12 *$ \\
\hline 4. Goal-setting & & & & -- & $.66^{* *}$ & $.45^{* *}$ & $-.14^{*}$ \\
\hline $\begin{array}{l}\text { 5. Competition } \\
\text { strategies }\end{array}$ & & & & & -- & $.57 * *$ & $-.26 * *$ \\
\hline 6. Positive rapport & & & & & & -- & $-.51 * *$ \\
\hline 7. Negative rapport & & & & & & & -- \\
\hline$M(S D)$ & 33.00 & 34.86 & 16.41 & 19.94 & 30.22 & 27.97 & 31.34 \\
\hline & $(9.26)$ & $(11.50)$ & $(7.61)$ & (9.38) & $(9.38)$ & $(9.55)$ & $(8.86)$ \\
\hline Skewness & -.43 & -.17 & .43 & .39 & -.15 & -.36 & .88 \\
\hline Kurtosis & -.39 & -.76 & -.62 & -.63 & -.55 & -.86 & .58 \\
\hline$\alpha$ & .87 & .94 & .93 & .95 & .90 & .93 & .82 \\
\hline
\end{tabular}

Note. ${ }^{*} p<.01,{ }^{* *} p<.001$ 


\section{ASSESSING COACHING BEHAVIOURS IN SPORT}

\section{Table 2.}

Standardized Solution of Factor Loadings, Error and Explained Variance $\left(R^{2}\right)$ for the CBS-S in Sample $1(n=499)$.

\begin{tabular}{lccccc}
\hline Factor & Item & Factor loadings & Standard error & $R^{2}$ & Error variance \\
\hline Physical training and planning & 1 & .82 & .02 & .67 & .33 \\
Physical training and planning & 2 & .74 & .03 & .55 & .65 \\
Physical training and planning & 3 & .78 & .02 & .61 & .39 \\
Physical training and planning & 4 & .78 & .02 & .61 & .39 \\
Physical training and planning & 5 & .67 & .03 & .46 & .54 \\
Physical training and planning & 6 & .77 & .03 & .59 & .41 \\
Physical training and planning & 7 & .72 & .04 & .52 & .48 \\
Technical skills & 8 & .88 & .01 & .77 & .23 \\
Technical skills & 9 & .91 & .01 & .83 & .17 \\
Technical skills & 10 & .80 & .02 & .63 & .37 \\
Technical skills & 11 & .92 & .01 & .85 & .15 \\
Technical skills & 12 & .76 & .02 & .58 & .42
\end{tabular}




$\begin{array}{llllll}\text { Technical skills } & 13 & .83 & .02 & .69 & .31 \\ \text { Technical skills } & 14 & .85 & .02 & .72 & .28 \\ \text { Technical skills } & 15 & .83 & .02 & .69 & .31 \\ \text { Goal setting } & 16 & .87 & .01 & .76 & .24 \\ \text { Goal setting } & 17 & .84 & .02 & .70 & .30 \\ \text { Goal setting } & 18 & .90 & .01 & .82 & .18 \\ \text { Goal setting } & 19 & .91 & .01 & .82 & .18 \\ \text { Goal setting } & 20 & .90 & .01 & .81 & .19 \\ \text { Mental preparation } & 21 & .92 & .01 & .84 & .16 \\ \text { Mental preparation } & 22 & .92 & .01 & .84 & .16 \\ \text { Mental preparation } & 23 & .89 & .01 & .79 & .21 \\ \text { Mental preparation } & 24 & .89 & .01 & .79 & .21 \\ \text { Mental preparation } & 25 & .87 & .01 & .76 & .24 \\ \text { Mental preparation } & 26 & .90 & .01 & .82 & .18 \\ \text { Competition strategies } & 27 & .89 & .01 & .79 & .21\end{array}$




$\begin{array}{llllll}\text { Competition strategies } & 28 & .85 & .02 & .71 & .29 \\ \text { Competition strategies } & 29 & .86 & .02 & .74 & .26 \\ \text { Competition strategies } & 30 & .76 & .02 & .58 & .42 \\ \text { Competition strategies } & 31 & .84 & .02 & .71 & .29 \\ \text { Competition strategies } & 32 & .73 & .02 & .53 & .47 \\ \text { Competition strategies } & 33 & .55 & .03 & .31 & .69 \\ \text { Personal rapport } & 34 & .91 & .02 & .82 & .18 \\ \text { Personal rapport } & 35 & .87 & .01 & .76 & .24 \\ \text { Personal rapport } & 36 & .89 & .01 & .79 & .21 \\ \text { Personal rapport } & 37 & .87 & .01 & .76 & .24 \\ \text { Personal rapport } & 38 & .89 & .01 & .79 & .21 \\ \text { Personal rapport } & 39 & .72 & .03 & .52 & .48 \\ \text { Negative personal rapport } & 40 & .40 & .05 & .16 & .84 \\ \text { Negative personal rapport } & 41 & .20 & .05 & .04 & .96 \\ \text { Negative personal rapport } & 42 & .86 & .03 & .74 & .26\end{array}$




\begin{tabular}{lccccc} 
Negative personal rapport & 43 & .89 & .02 & .78 & .22 \\
Negative personal rapport & 44 & .48 & .05 & .23 & .77 \\
Negative personal rapport & 45 & .72 & .04 & .52 & .48 \\
Negative personal rapport & 46 & .74 & .04 & .54 & .46 \\
Negative personal rapport & 47 & .79 & .03 & .62 & .38 \\
\hline
\end{tabular}


Table 3.

Pearson Product Moment Correlations of Test-Retest Reliability (Sample 2; $n=39$ ) and Concurrent Validity (Sample 1; n=495) of the CBS-S.

\begin{tabular}{|c|c|c|c|c|c|c|c|c|}
\hline & 1. CBS-S & 2. CBS-S & 3. CBS-S & 4. CBS-S & 5. CBS-S & 6. CBS-S & 7. CBS-S & \\
\hline & Physical & Technical & Mental & Goal- & Compe- & Personal & Negative & \\
\hline & training & skills & preparation & setting & tition & rapport & personal & \\
\hline & and & & & & Strategies & & rapport & \\
\hline Assessment & planning & & & & & & & $\alpha$ \\
\hline \multicolumn{9}{|c|}{ Test-retest $(n=39)$} \\
\hline CBS-S & $.82 * *$ & $.64 * *$ & $.58 * *$ & $.66^{* *}$ & $.59 * *$ & $.78 * *$ & $.75 * *$ & -- \\
\hline \multicolumn{9}{|c|}{ Concurrent validity ( $n=495)$} \\
\hline LSS: Training & $.34 * *$ & $.65 * *$ & $.56 * *$ & $.60 * *$ & $.71 * *$ & $.57 * *$ & $-.17 * *$ & .87 \\
\hline \multicolumn{9}{|l|}{ and instruction } \\
\hline LSS: & $.16 * *$ & $.35 * *$ & $.40 * *$ & $.82 * *$ & $.53 * *$ & $.55^{* *}$ & $-.33 * *$ & .81 \\
\hline
\end{tabular}


behaviour

$\begin{array}{llllllllll}\text { LSS: Social } & .17^{* *} & .43^{* *} & .52^{* *} & .84^{* *} & .57^{* *} & .69^{* *} & -.25^{* *} & .77\end{array}$

support

$\begin{array}{lllllllll}\text { LSS: Positive } & .13 * & .44 * * & .42 * * & .68 * * & .52 * * & .57 * * & -.34 * * & .83\end{array}$

feedback

$\begin{array}{lllllllll}\text { CSAI-2R: Self- } & .08 & .24 * * & .28^{* *} & .22^{* *} & .28^{* *} & .29 * * & -.21^{* *} & .89\end{array}$

confidence

$\begin{array}{lllllllll}\text { CART-Q: } & .21 * * & .54 * * & .44 * * & .50 * * & .61 * * & .78^{* *} & -.44^{* *} & .95\end{array}$

Note. ${ }^{*} p<.05, * * p<.01$ 